\title{
Multi-objective Genetic Optimization for LCLS-II X-ray
}

\section{FEL}

\author{
Lanfa Wang and Tor O. Raubenheimer
}

SLAC (Stanford Linear Accelerator Center), Stanford University, California 94025, USA

Received: May 09, 2014 / Accepted: May 31, 2014 / Published: August 25, 2014.

\begin{abstract}
The LCLS-II (linac coherent light source II) will build on the success of the world's most powerful X-ray laser: the LCLS (linac coherent light source). It will add two new X-ray laser beams and room for additional new instruments, thus greatly increasing the number of experiments carried out each year. Multiple operation modes are proposed to accommodate a variety of user requirements. There are a large number of variables and objectives in the design. For each operation mode, MOGA (multi-objective genetic algorithm) is applied to optimize the machine parameters in order to minimize the jitters, energy spread, collective effects and emittance.
\end{abstract}

Key words: MOGA, optimization, FEL (free-electron laser).

\section{Introduction}

LCLS-II (linac coherent light source II) will provide beam with difference charge. For each beam, the $R 56$ at $B C 1$ (bunch compressor 1) and $B C 2$ (bunch compressor 2), accelerating structure phase and voltage are optimized using MOGA (multi-objective genetic algorithm) program to satisfy the required peak current, and to minimize the energy spread, energy chirp, current jitter, energy jitter and time jitter. We briefly summarize the MOGA optimization for LCLS (linac coherent light source), LCLS-II and the two beam configurations.

The transverse emittance growth due to CSR (coherent synchrotron radiation) is minimized by choosing appropriate phase advance between $B C 2$ and the downstream bending magnets. The final emittance at the beginning of the undulator is just about $1 \mathrm{um}$ and even lower for low charge.

The paper is organized as follows: Section 2 benchmarks with the LCLS beam; Section 3 discusses the optimization for LCLS-II; Section 4 introduces the

\footnotetext{
Corresponding author: Wang Lanfa, physicist, Ph.D., research field: particle accelerator. E-mail: wanglf@slac.stanford.edu.
}

possibility of LCLS-II with two beam energies; Section 5 discusses the minimization of CSR induced emittance growth; and Section 6 gives conclusions.

\section{Benchmark with LCLS Beam}

It is important to have a comparision of the simulation with the measurements. A series of data, for instance, the voltage and phase of $L 1$ (Linac 1) and $L 2$ (Linac 2), energy at $B C 1, B C 2$ and $D L 2$ (dog legs), beam current at $B C 1$ and $B C 2$, were taken at the LCLS to set up the variations and then compare the jitters in beam current and energy. One example of the variation of $L 2$ voltage and $D L 2$ energy is shown in Fig. 1. We use these varations to study the enegy and beam current jitter. The main machine parameters used in the simulation are listed in Table 1 as operational model. The values of these parameters are not exactly the same as the readings from MCC (main control center). Some parameters, especially the phase of RF (radio frequency), are tweaked to get flat top current profile and zero energy chirp at the beginning of the undulator similar to the measured values. The bunch charge is 150 pC. Fig. 2 shows the bunch profile before the undulator by LiTrack simulation, 
which gives a simliar bunch current $\sim 3 \mathrm{kA}$. The measured energy jitter in the machine is about $0.049 \%$, which is slighly larger than the the simulation result of $0.033 \%$. The $L 0$ jitter is not included in the simulation since the simulation starts after $L 0$ and this will cause the simulated jitter to be low [1].

The main contributions of the energy jitters in the operational mode are $L 1$ phase and voltage, $L X$ (X-band Linac) phase and $L 2$ phase. One of the optimized configurations is also listed in Table 1. This

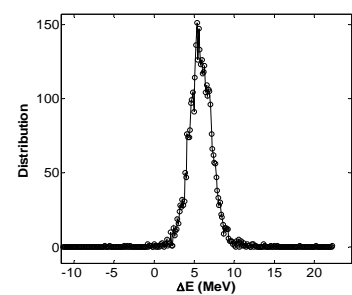

(a)

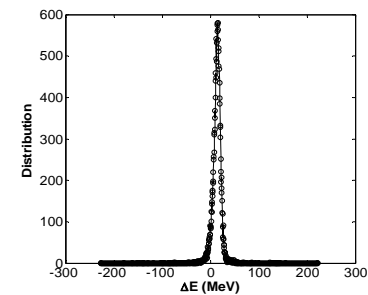

(b)
Fig.1 Variation of the (a) $L 2$ voltage and (b) $D L 2$ energy at LCLS.

Table 1 Example of LCLS operational and optimized model.

\begin{tabular}{lll}
\hline Variables & Optimized & operational \\
\hline$I_{p k}(\mathrm{kA})$ & 3 & $\sim 3$ \\
$\phi_{L 1}($ degree $)$ & -19.3 & $\sim-26.1$ \\
$V_{L 1}(\mathrm{MeV})$ & 111 & $\sim 118$ \\
$\phi_{L \mathrm{x}}($ degree $)$ & -154 & $\sim-160$ \\
$V_{L \mathrm{x}}(\mathrm{MeV})$ & 22 & $\sim 22$ \\
$\phi_{L 2}(\mathrm{degree})$ & -19 & $\sim-38.7$ \\
$V_{L 2}(\mathrm{GeV})$ & 5.06 & $\sim 6.15$ \\
$\phi_{L 3}(\mathrm{degree})$ & -10.3 & $\sim 0$ \\
$V_{L 3}(\mathrm{GeV})$ & 8.79 & $\sim 7.667$ \\
$R_{56} @ B C 1(\mathrm{~mm})$ & -45.5 & $\sim-45.5$ \\
$R_{56} @ B C 2(\mathrm{~mm})$ & -51.3 & $\sim-20.6$ \\
$(\Delta I / I)(\%)$ & 10 & $\sim 7$ \\
$(\Delta E / E)(\%)$ & 0.014 & $\sim 0.033$ \\
\hline
\end{tabular}

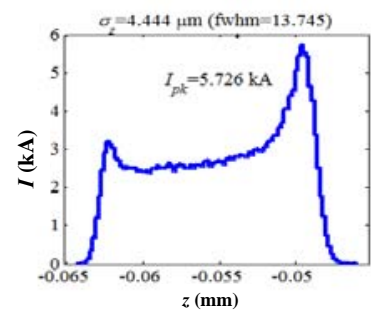

(a)

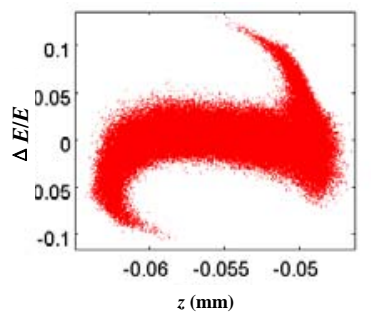

(b)
Fig. 2 The bunch profile and phase space at the beginning of the undulator for $150 \mathrm{pC}$ beam at LCLS. Bunch head is to the left.

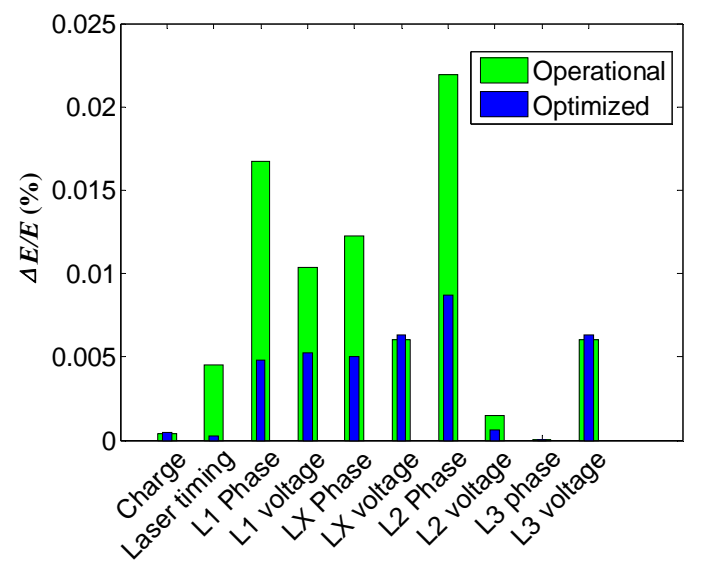

Fig. 3 Comparison of the distribution of energy jitters for the operational mode and optimized one.

optimized configuration reduces the energy jitter by a factor 2. Fig. 3 shows the comparision of the energy jitter for the optimized mode and the operational one. There are large reductions for the four major contributions. The energy jitter of the optimized mode is widely distributed compared with the operational mode. It clearly shows the benefit from optimization. We are doing detailed benchmark with the measurement: taking the OTR4 phase space data as the input of the simulation and comparing the phase space in the middle of $B C 1, B C 2$ and $D L 2$. New features are being added to Litrack code for such comparision.

\section{Optimization of LCLS-II}

Fig. 4 shows the layout of LCLS-II design. The energy at the $B C 1$ and $B C 2$ is $335 \mathrm{MeV}$ and $4.5 \mathrm{GeV}$, respectively. The Gun simulation is done using IMPACT. The Litrack code is used to study the longitudinal dynamics from $L 0$ to the beginning of undulator. There are 10 variables in the optimization: the phases and voltages of $L 1, L X, L 2, L 3$ (Linac 3), $R 56$ at $B C 1$ and $B C 2$. The constraints include the energies at $B C 1, B C 2$ and beginning of the undulator, the cancellation of quadratic energy chirp with $\mathrm{X}$-band structure, and a peak current of $3.0 \mathrm{kA}$ or $4.0 \mathrm{kA}$ at the end of beam line. For each bunch charge mode, we need to minimize the energy spread, linear energy 


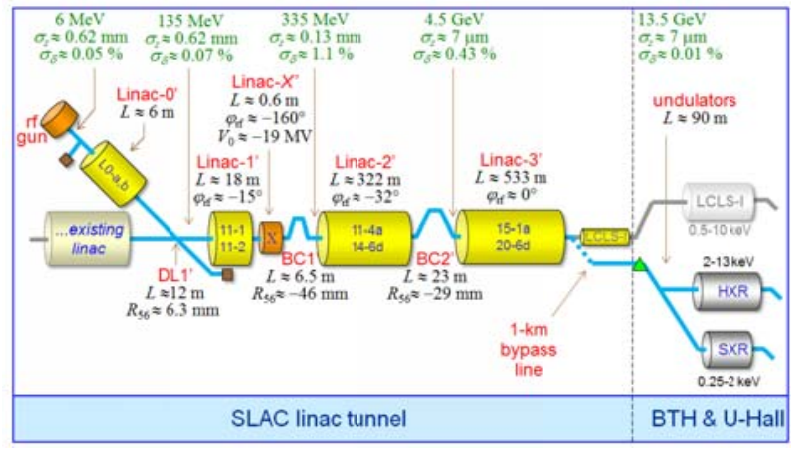

Fig. 4 Layout of LCLS-II.

chirp, peak current jitter, energy jitter and timing jitter. All these jitters are normalized and added together according to their weights to get a single objective. In most of the optimization, we set an equal weight for them. The resistive wall wake of the chamber and the wake field of the accelerating structures are also included in the simulation.

The optimization is done for each bunch charge. Table 2 shows the example of the solutions for $250 \mathrm{pC}$ and $150 \mathrm{pC}$ cases. These solutions have smaller timing and energy jitters than required for SASE operation while the current jitter is close to the requirement of $12 \%$. In the optimization, an equal weight is used for energy, current and timing jitters. We can set a larger weight for the current jitter to reduce the current jitter, if desired.

Fig. 5 shows the phase space along the beam line for $250 \mathrm{pC}$ case. It clearly shows a non-zero energy chirp at the end of the linac (L3) and the subsequent reduction of the chirp before the undulator due to the wake field effect. Fig. 6 shows the current profile of $150 \mathrm{pC}$ case. There is a double horn in the beam profile due to the effect of nonlinear wake field. Fig. 7 shows the distribution of the jitters for $250 \mathrm{pC}$ case. The variations (errors) used for the jitter study are listed in Table 3. The jitters are widely distributed, especially for the energy jitter. The current jitter is dominant by the effect of $L X$ phase, $L 2$ phase and $L 1$ voltage, the timing jitter is not an issue. The energy jitter is important for the seeding FEL (free-electron laser) in LCLS. An energy jitter of $0.042 \%$, which is close to the measured energy jitter in LCLS, reduces the FEL intensity to $70 \%$ of the peak value. If the energy jitter can be reduced to $0.02 \%$, the FEL intensity will be $90 \%$ of the peak. We are working to minimize the energy jitter in various ways, including MOGA optimization.

\section{LCLS-II+, Two Beam Energy Machine}

We present one potential upgrade to the LCLS-II design, referred to as LCLS-II+. This option integrates LCLS and LCLS-II together to provide two simultaneous beam energies at a $360 \mathrm{~Hz}$ repetition rate. Fig. 8 shows the sketch of LCS-II+. The LCLS-II linac, operated at $360 \mathrm{~Hz}$, can provide low energy

Table 2 Configurations and jitters of different bunch charge.

\begin{tabular}{lll}
\hline Variables & $250 \mathrm{pC}$ & $150 \mathrm{pC}$ \\
\hline$I_{p k}(\mathrm{kA})$ & 3 & 3 \\
$\phi_{L 1}(\mathrm{degree})$ & -26 & -24 \\
$V_{L 1}(\mathrm{MeV})$ & 262 & 248 \\
$\phi_{L \mathrm{x}}(\mathrm{degree})$ & -165 & -168 \\
$V_{L \mathrm{x}}(\mathrm{MeV})$ & 38 & 29 \\
$\phi_{L 2}(\mathrm{degree})$ & -38 & -36 \\
$V_{L 2}(\mathrm{GeV})$ & 5.26 & 5.16 \\
$\phi_{L 3}(\mathrm{degree})$ & -4 & -3 \\
$V_{L 3}(\mathrm{GeV})$ & 9.06 & 9.04 \\
$R_{56} @ B C 1(\mathrm{~mm})$ & -30 & -38 \\
$R_{56} @ B C 2(\mathrm{~mm})$ & -22 & -23 \\
$\sigma_{E} / E(\%)$ & 0.013 & 0.013 \\
$(\Delta I / I)(\%)$ & 9 & 14 \\
$(\Delta E / E)(\%)$ & 0.039 & 0.038 \\
$(\Delta \tau)(\mathrm{fs})$ & 49 & 51 \\
\hline
\end{tabular}
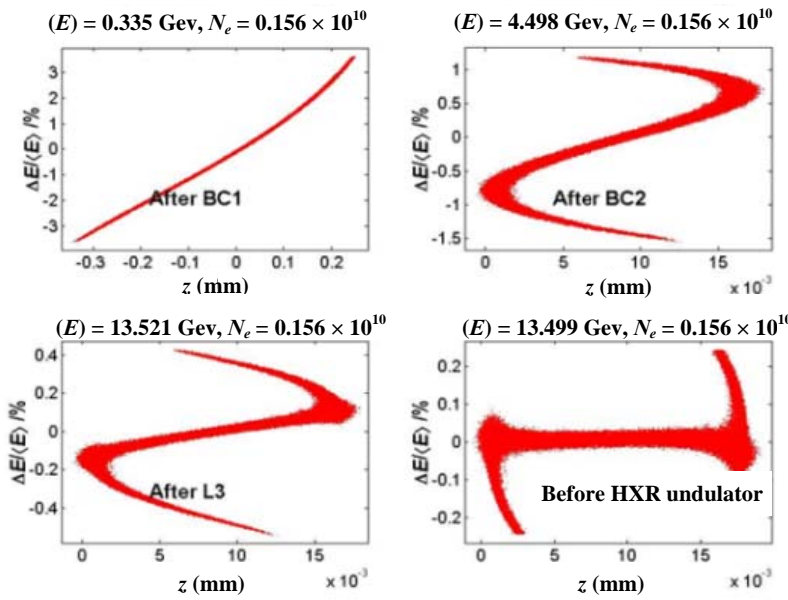

Fig. 5 Example of phase space for $250 \mathrm{pC}$ Hard X-ray at different locations along the linac: $B C 1, B C 2, L 3$ and undulator beginning. 

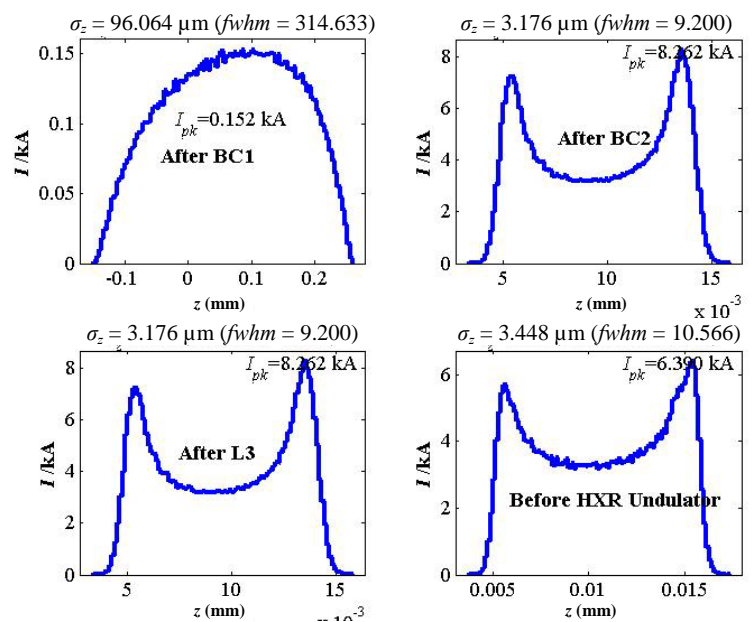

Fig. 6 Example of beam profile for $150 \mathrm{pC}$ hard X-ray at different locations along the Linac.

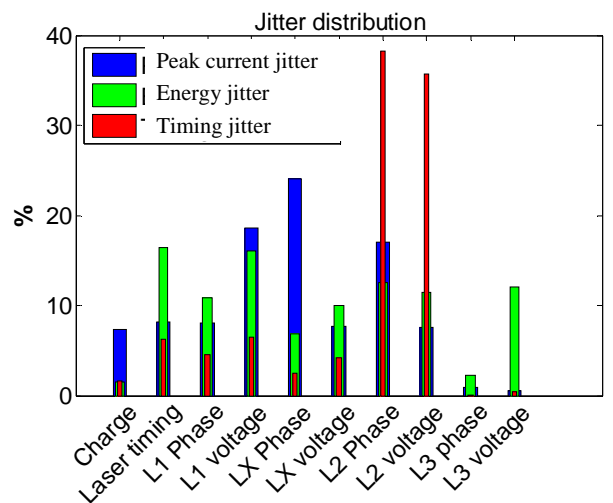

Fig. 7 Distributions of the jitters for 250 pC hard X-ray configuration.

Table 3 The tolerance used for evaluation of the jitters.

\begin{tabular}{lll}
\hline & Symbol & errors \\
\hline Relative bunch charge & $\Delta \mathrm{Q} / \mathrm{Q}$ & $1 \%$ \\
Driven laser timing & $\Delta \tau$ & $0.2 \mathrm{ps}$ \\
$L 1$ RF phase & $\Delta \phi_{1}$ & $0.05^{\circ}$ \\
$L X$ RF phase & $\Delta \phi_{\mathrm{x}}$ & $0.3^{\circ}$ \\
$L 2$ RF phase & $\Delta \phi_{2}$ & $0.04^{\circ}$ \\
$L 3$ RF phase & $\Delta \phi_{3}$ & $0.03^{\circ}$ \\
$L 1$ RF relative voltage & $\Delta \mathrm{V} / \mathrm{V}_{1}$ & $0.05 \%$ \\
$L X$ RF relative voltage & $\Delta \mathrm{V} / \mathrm{V}_{\mathrm{x}}$ & $0.25 \%$ \\
$L 2$ RF relative voltage & $\Delta \mathrm{V} / \mathrm{V}_{2}$ & $0.05 \%$ \\
$L 3$ RF relative voltage & $\Delta \mathrm{V} / \mathrm{V}_{3}$ & $0.02 \%$ \\
\hline
\end{tabular}

beam, for instance $7.5 \mathrm{GeV}$. Some low energy bunches (after $L 3$ ) are kicked to the LCLS-II by-pass beam line to radiate directly. The rest of the low energy bunches are continuously accelerated and compressed along the existing LCLS accelerator to achieve an energy above $16 \mathrm{GeV}$. The first bunch compressor in LCLS is replaced by a BL (bunch lengthener) to increase the bunch length in order to increase the energy chirp and also reduce the effect of wake field. The combination of $\mathrm{BL}$ and $B C 3$ provide the flexibility to adjust the bunch current/profile of the high energy beam. A wake field type of de-chirper [2] could be added in the bypass line as an option to control the final energy chirp. It is not used in this design.

To increase the repetition rate from current $120 \mathrm{~Hz}$ to $360 \mathrm{~Hz}$, the maximum accelerating gradient is lower by a factor of $\sim 1.8$. Therefore, a longer accelerating structure is needed to get the same beam energy. The existing S-band accelerating structure is assumed for $360 \mathrm{~Hz}$ repetition rate. X-band can be chosen for even high repetition rate. However, the stronger wake field may limit the flexibility to achieve desired beam and it is also expensive.

It is import to study the flexibility to provide the two simultaneous beams with good beam quality, such as high peak current with small energy spread, for different charges. Fig. 9 shows the example of 150 pC bunch charge case. Both beams have high peak current, above $3 \mathrm{kA}$, and small energy chirp. A small positive energy chirp is intentionally kept for the low energy beam, which can be easily adjusted by the adjusting the RF phases. There are small $R 56$ of $3.5 \mathrm{~mm}$ at BL and $-7.5 \mathrm{~mm}$ at $B C 3$. The peak current can be easily adjusted by the change of $R 56$ at $B C 3$ and BL.

Fig. 10 shows the $20 \mathrm{pC}$ charge case. The $R 56$ is 2.5 $\mathrm{mm}$ and $-6.0 \mathrm{~mm}$ at $\mathrm{BL}$ and $B C 3$, respectively. Again, there is large flexibility to adjust the peak current of the high energy beam. The peak current is about $4 \mathrm{kA}$ in the example. The energy chirps for both beams are about zero. Study shows that high bunch charge 250 $\mathrm{pC}$ also works well. The large flexibilities of this two energy scheme with high repetition rate of $360 \mathrm{~Hz}$ make this type of machine very attractive.

\section{Minimization of Emittance Growth due to CSR}

The collective effects, i.e., SC (space charge) forces, geometric wake fields in the accelerating structures 


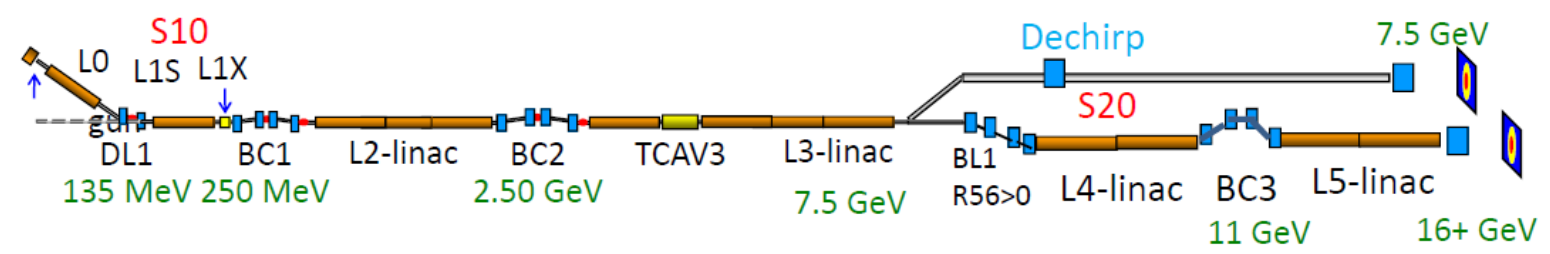

Fig. 8 Sketch of LCLS-II+, a two beam energy FEL machine beyond LCLS-II.
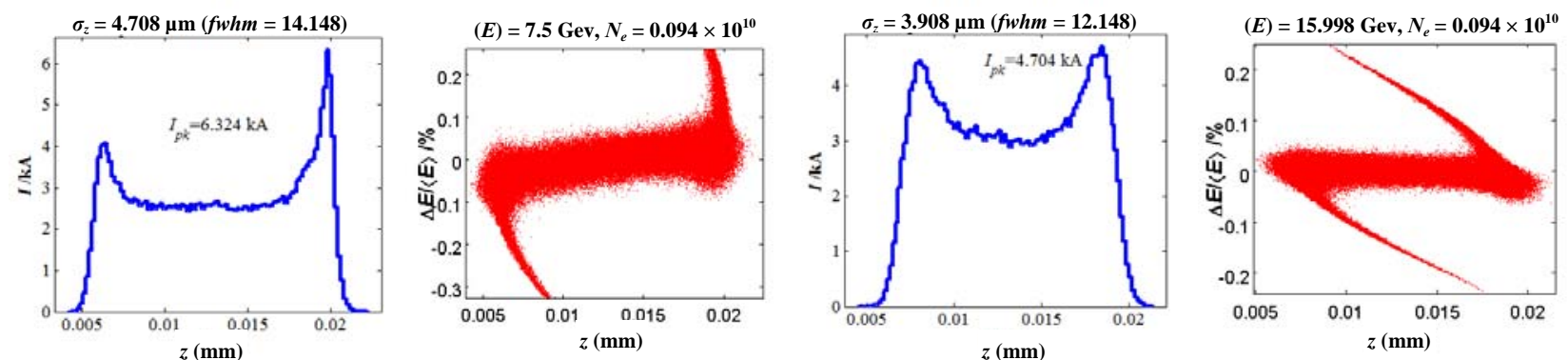

Fig. 9 The bunch profile and phase space of low energy beam (top) and high energy beam before the undulator for 150pC beam. Bunch head is on the left.
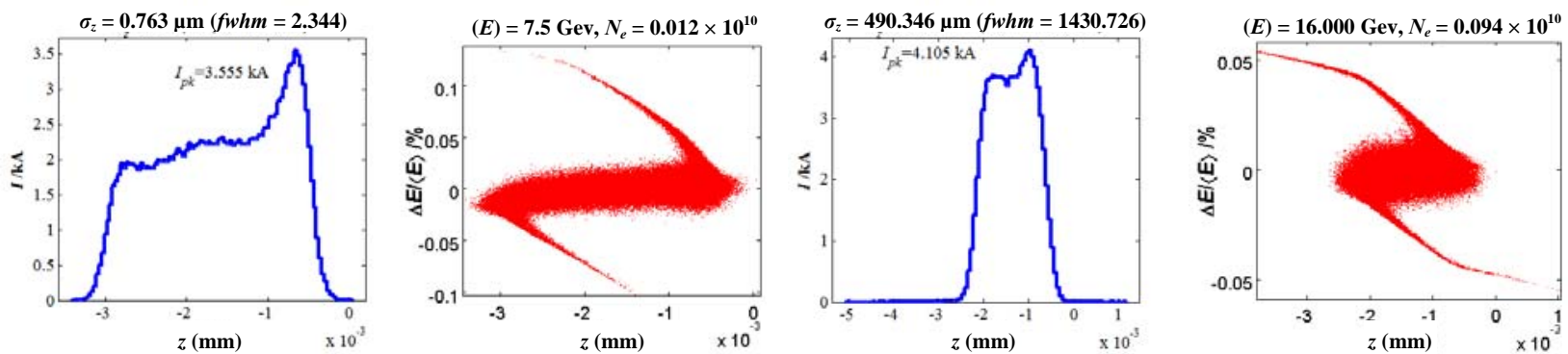

Fig. 10 The bunch profile and phase space of low energy beam (top) and high energy beam before the undulator for $20 \mathrm{pC}$ beam.

and the CSR emission in dispersive systems, can induce projected emittance growth. Among them, the CSR effect can be minimized by a better design. The energy modulation and transverse emittace excitation induced by CSR in different dispersion sections can be cancelled [3-6] or moderated with an appropriated design of the optics.

There are four dispersion sections in LCLS-II, two bunch compressors, DL2 and HBEND section. Fig. 11 shows the Twiss functions and dispersion along the hard X-ray beam line. Since both beam and optics are different in these dispersion sections, there is no perfect cancellation of CSR effect. To minimize the final transverse emittance before the undulator, a virtual phase shifter before HBEND dispersion section is added. Furthermore, we assume that both the horizontal and vertical phase can be adjusted independently. The simulations have been done with elegant code [7]. Fig. 12 shows the dependence of the emittance at the beginning of the undulator on the phase shift for the case of $250 \mathrm{pC}$ charge hard X-ray beam. There is a maximum horizontal emittance of 3.4

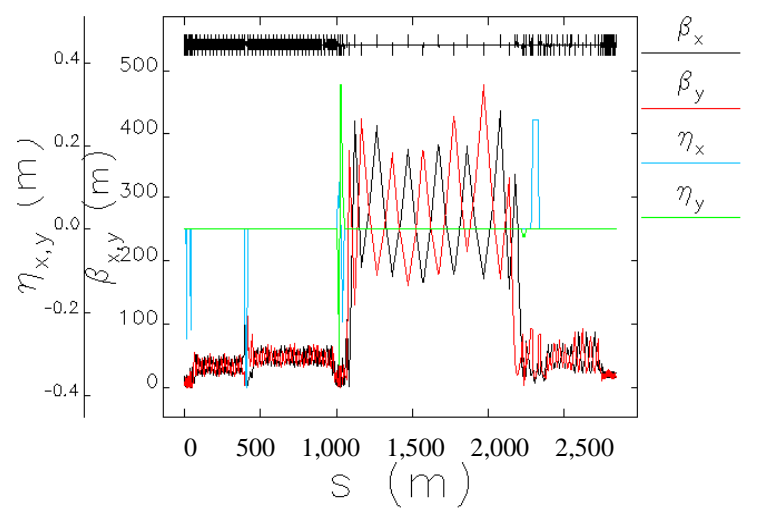

Fig. 11 Twiss functions and dispersion along the hard X-ray beam line. 


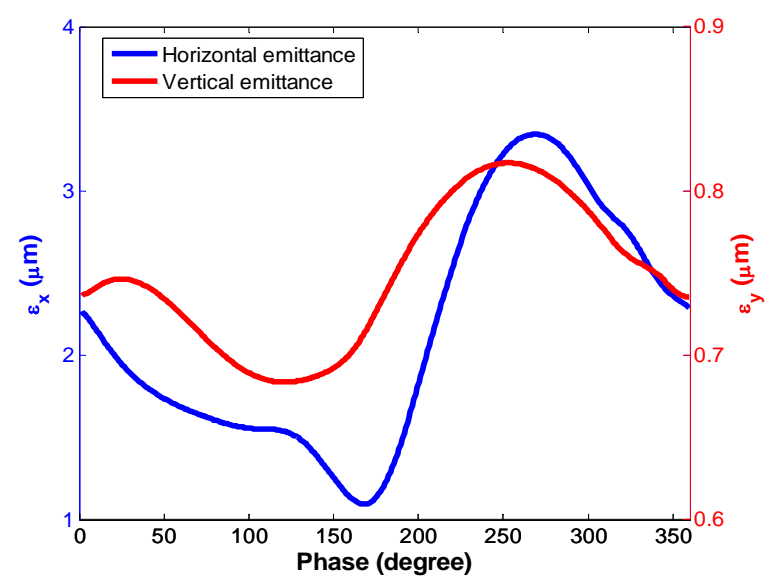

Fig. 12 Effect of phase shift on the emittace at the beginning of the undulator for $250 \mathrm{pC}$ hard $\mathrm{X}$-ray beam.

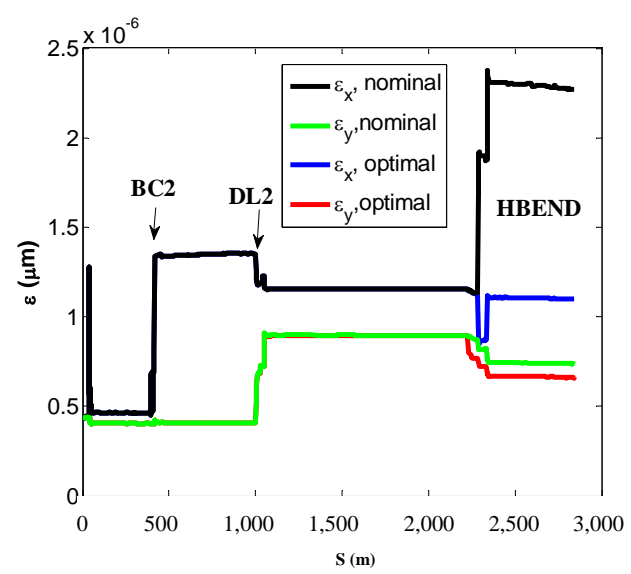

Fig. 13 The growth of emittance along the hard X-ray beam line with $250 \mathrm{pC}$ beam.

$\mu \mathrm{m}$ and a minimum one of $1.09 \mu \mathrm{m}$ at $167.5^{\circ}$. While there is weaker dependence of the vertical emittance on the vertical phase as expected. Fig. 13 shows the growth of the projected emittance along the LCLS-II beam line. The cancellation is clear seen with the optimized solution. Studies with different charge show the optimized phases are slightly different.

Besides the phase advance, the $H$-function $\left(H=\eta^{2}+\left(\beta \eta^{\prime}+\alpha \eta\right)^{2} / \beta\right)$ of the bunch compressor can be minimized to reduce the CSR effect [8]. More complicated compressors which offer opposite signs of the dispersions [9] can provide better compensation.

\section{Summary}

MOGA is applied to optimize the LCLS-II, LCLS and LCLS-II+ in order to minimize the jitters, energy spread and energy chirp. Small energy spread, zero energy chirp and small jitter are achieved for different bunch charge. MOGA provides a very useful tool in the design. Our preliminary study shows that the energy jitter in LCLS can be reduced by a factor of 2 by optimizing the machine configuration. LCLS-II+ can provide two beams with different energies simultaneously and large flexibilities in beam energy, bunch charge and energy chirp.

The emittance growth due to CSR can be minimized by simply choosing an appropriate phase advance between $B C 2$ and DL2 or LTU. The optimal horizontal emittance is about $1.1 \mu \mathrm{m}$ and $0.3 \mu \mathrm{m}$ for $250 \mathrm{pC}$ and $40 \mathrm{pC}$ case. The emittance can be further minimized by reducing the betatron function at $B C 2$.

An integrated S2E (start-to-end) optimization is desired to further optimize the injector and undulator.

\section{Acknowledgments}

The author wishes to thank F. Zhou, M. Woodley, Y. Nosochkov, J. Wu, Y. Ding, Z. Huang, F. J. Decker, A. Krasnykh, J. Welch, J. Turner, T. Maxwell and LCLS Operation Team for discussion and help. This work is supported by Department of Energy Contract No. DE-AC02-76SF00515

\section{References}

[1] Decker, F. J. 2013. "Increased Stability Requirements for Seeded Beams at LCLS." Presented at the 35th International Free-Electron Laser (FEL 2013), New York, USA.

[2] Emma, P. 2014. "Experimental Demonstration of Energy-Chirp Control in Relativistic Electron Bunches Using a Corrugated Pipe.” Phys. Rev. Lett. 112 (January): 034801.

[3] Mitri, S. D. 2013. "Cancellation of Coherent Synchrotron Radiation Kicks with Optics Balance.” Phys. Rev. Lett. 110 (January): 014801.

[4] Douglas, D. 1998. Suppression and Enhancement of CSR-Driven Emittance Degradation in the IR-FEL Driver. Thomas Jefferson national accelerator facility report.

[5] Courant, E. D., and Snyder, H. S. 1958. "Theory of the Alternating-Gradient Synchrotron.” Ann. Phys. 3 (1): 
360-408.

[6] Lee, S. Y. 2007. Accelerator Physics. Singapore: World Scientific.

[7] Borland, M. 2000. Elegant: A Flexible SDDS-Compliant Code for Accelerator Simulation, APS LS-287

[8] Mitri, S. D., and Cornacchia, M. 2014. "Merit
Functions for the Linac Optics Design for colliders and Light Sources.” Nucl. Instrum. Methods A 735: 60-5.

[9] Yichao, J., Yue, H., and Vladimir, N. L. 2013. "Compensating Effect of the Coherent Synchrotron Radiation in Bunch Compressors.” Phys. Rev. ST Accel. Beams 16 (June): 060704. 